\title{
Estimation of Functional Ranges Using Standard and Inner Interval Arithmetic
}

\author{
Julius ŽILINSKAS \\ Institute of Mathematics and Informatics \\ Akademijos 4, 08663, Vilnius, Lithuania \\ e-mail: julius.zilinskas@mii.lt
}

Received: January 2005

\begin{abstract}
New ways to estimate ranges of values of functions from standard and inner interval arithmetic have been proposed. Using the proposed ways ranges of values of mathematical test functions for global optimization and of objective functions for practical global optimization problems have been estimated and compared. Results of the experiments show that it is promising to use proposed balanced interval arithmetic in interval global optimization.
\end{abstract}

Key words: interval arithmetic, random interval arithmetic, interval global optimization.

\section{Introduction}

Interval global optimization methods are based on interval arithmetic proposed in (Moore, 1966). The lower and upper bounds for values of function in the sub-region are evaluated applying the interval operations with intervals instead of the real operations with real variables in the algorithm of calculation values of function. The bounds are useful to detect the sub-regions of the feasible region which cannot contain a global minimizer. Such bounds are used in branch and bound algorithms for global optimization. A disadvantage of standard interval arithmetic is the dependency problem which causes widening of computed intervals and slowing of optimization.

Balanced random interval arithmetic proposed in (Žilinskas and Bogle, 2004) extending the ideas of (Alt and Lamotte, 2001), is obtained by choosing standard and inner interval operations at each step of the computation randomly with the predefined probabilities of standard and inner operations. The disadvantages of balanced random interval arithmetic are that estimation of ranges with this arithmetic uses more calculations than with standard interval arithmetic and that it is based on the assumption that distributions of centres and radii of the evaluated random intervals are normal and folded normal (Johnson and Kotz, 1994-1995) (also known as absolute normal, because radii cannot be negative) respectively. To overcome the disadvantages of balanced random interval arithmetic, new ways to estimate ranges of values of functions based on interval arithmetic are proposed in this paper.

Using the proposed ways ranges of values of mathematical test functions for global optimization and of objective functions for practical global optimization problems have 
been estimated and compared. Mathematical test functions for global optimization known from literature, and objective functions for practical global optimization problems (multidimensional scaling and separation problem) are used in our experiments. Most of the used functions are defined in (Madsen and Žilinskas, 2000). The function of separation problem is defined in (Csendes, 1998).

\section{Interval Arithmetic}

Interval arithmetic has been proposed in (Moore, 1966). Interval arithmetic operates with real intervals $\underline{\bar{x}}=[\underline{x}, \bar{x}]=\{x \in \Re \mid \underline{x} \leqslant x \leqslant \bar{x}\}$, defined by two real numbers $\underline{x} \in \Re$ and $\bar{x} \in \Re, \underline{x} \leqslant \bar{x}$. For any real arithmetic operation $x \circ y$ the corresponding interval arithmetic operation $\underline{x} \circ \bar{y}$ is defined whose result is an interval containing every possible number produced by real operation with real numbers from each interval. The standard interval arithmetic operations are defined as:

$$
\begin{aligned}
& \underline{\bar{x}}+\underline{\bar{y}}=[\underline{x}+\underline{y}, \bar{x}+\bar{y}], \\
& \underline{\bar{x}}-\underline{\bar{y}}=[\underline{x}-\bar{y}, \bar{x}-\underline{y}], \\
& \underline{\bar{x}} \times \underline{\bar{y}}= \begin{cases}{[\underline{x} \underline{y}, \overline{x y}],} & \underline{\bar{x}}>0, \overline{\bar{y}}>0, \\
{[\bar{x} \underline{y}, \overline{x y}],} & \underline{\bar{x}}>0, \overline{\bar{y}} \ni 0, \\
{[\bar{x} \underline{y}, \underline{x} \bar{y}],} & \underline{\bar{x}}>0, \overline{\bar{y}}<0, \\
{[\underline{x} \bar{y}, \overline{x y}],} & \underline{\bar{x}} \ni 0, \overline{\bar{y}}>0, \\
{[\min (\underline{x} \bar{y}, \bar{x} y), \max (\underline{x} \underline{y}, \overline{x y})],} & \underline{\bar{x}} \ni 0, \overline{\bar{y}} \ni 0, \\
{[\bar{x} y, \underline{x} y],} & \underline{\bar{x}} \ni 0, \overline{\bar{y}}<0, \\
{[\underline{x} \bar{y}, \bar{x} \underline{y}],} & \underline{\bar{x}}<0, \overline{\bar{y}}>0, \\
{[\underline{x} \bar{y}, \underline{x} \underline{y}],} & \underline{\bar{x}}<0, \overline{\bar{y}} \ni 0, \\
{[\overline{x y}, \underline{x} \underline{y}],} & \underline{\bar{x}}<0, \underline{\bar{y}}<0,\end{cases}
\end{aligned}
$$

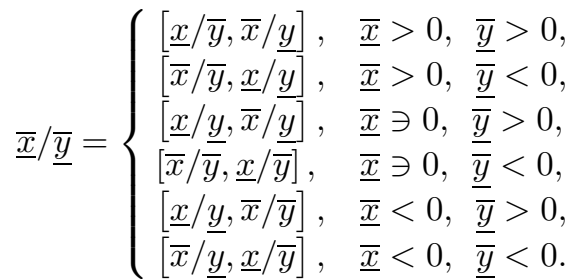

The guaranteed lower and upper bounds for values of function in the region defined by intervals of variables can be evaluated applying standard interval operations with intervals instead of real operations in the algorithm to calculate the values of function. The evaluated bounds always enclose all values of function in the defined region:

$$
\left\{f(X) \mid X \in \underline{\bar{X}}, \underline{X} \in \Re^{n}, \bar{X} \in \Re^{n}\right\} \subseteq \underline{\bar{f}}(\underline{\bar{X}}),
$$

where $f: \Re^{n} \rightarrow \Re, \underline{f}:[\Re, \Re]^{n} \rightarrow[\Re, \Re]$. 
The bounds may be used in global optimization to detect the sub-regions of the feasible region not containing a global minimizer. Such sub-regions may be discarded from the further search.

A disadvantage of standard interval arithmetic is the dependency problem (Hansen and Walster, 2003): when a given variable occurs more than once in interval computation, it is treated as a different variable in each occurrence. This causes widening of computed intervals.

For example, if

$$
f(X)=\sum_{i} x_{i}
$$

and

$$
f_{k}(X)=x_{k},
$$

then

$$
\underline{\bar{f}}(\underline{\bar{X}})-\underline{\overline{f_{k}}}(\underline{\bar{X}})=\sum_{i} \underline{\overline{x_{i}}}-\underline{\overline{x_{k}}}
$$

overestimates

$$
\left\{f(X)-f_{k}(X) \mid X \in \underline{\bar{X}}\right\}=\sum_{i \neq k} \underline{\overline{x_{i}}} .
$$

Therefore algorithms

$$
\begin{aligned}
& \begin{array}{l}
f=0 ; \\
\text { for } i=1 \text { to } N \\
f=f+x(i) ; \\
\text { for } k=1 \text { to } N \\
\text { some_function }(f-x(k)) \text {; } \\
\text { and } \\
\text { for } k=1 \text { to } N \\
f=0 ; \\
\text { for } i=1 \text { to } k-1 \\
\quad f=f+x(i) ; \\
\text { for } i=k+1 \text { to } N \\
\quad f=f+x(i) ; \\
\text { some_function (f); }
\end{array}
\end{aligned}
$$

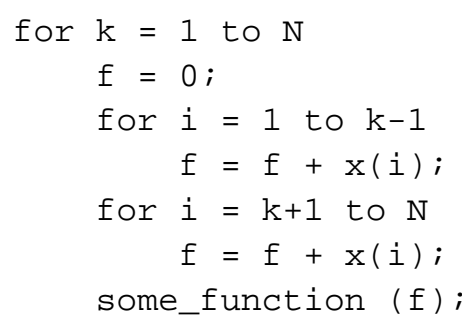

are not the same. The first is preferable by programmers because of speed of computations. The second is preferable by interval analysts because of tighter intervals. However dependency cannot be always avoided.

To get an idea how close is overestimation to the exact range of values of function guaranteed inner estimate (underestimate) is proposed in (Kreinovich et al., 1996). Other underestimating in interval computations is proposed in (Žilinskas and Žilinskas, 2005b) assuming some regularity of the dependency between variables. Inner interval arithmetic 
used in (Alt and Lamotte, 2001; Žilinskas and Bogle, 2003; Žilinskas and Bogle, 2004) gives so called inner approximation for the range.

Inner interval arithmetic assumes that all operands are dependent and exchange the ends of one operand in every operation

$$
\underline{\bar{x}} \circ_{\text {in }} \underline{\bar{y}}=[\underline{x}, \bar{x}] \circ[\bar{y}, \underline{y}],
$$

for example

$$
\begin{aligned}
& \underline{\bar{x}}+{ }_{\text {in }} \underline{\bar{y}}=[\underline{x}+\bar{y}, \bar{x}+\underline{y}], \\
& \underline{\bar{x}}-{ }_{\text {in }} \overline{\bar{y}}=[\underline{x}-\underline{y}, \bar{x}-\bar{y}], \\
& \underline{\bar{x}} \times_{\text {in }} \overline{\underline{y}}=[\underline{x} \bar{y}, \bar{x} \underline{y}, \quad \underline{\bar{x}}>0, \bar{y}>0, \\
& \underline{\bar{x}} /_{\text {in }} \underline{\bar{y}}=[\underline{x} / \underline{y}, \bar{x} / \bar{y}], \quad \underline{\bar{x}}>0, \underline{\bar{y}}>0 .
\end{aligned}
$$

If the ends of the resulting interval $\underline{\underline{x}} \circ_{i n} \underline{\bar{y}}>\underline{\bar{x}} \circ_{i n} \underline{\bar{y}}$, they are exchanged.

Using inner interval arithmetic

$$
\sum_{i} \underline{\overline{x_{i}}}-{ }_{i n} \underline{\overline{x_{k}}}=\sum_{i \neq k} \underline{\overline{x_{i}}} .
$$

However in the general case the evaluated inner interval is tighter than the full range of values of function. Moreover usually dependency and function of operands are not known when arithmetic operation should be performed, therefore it is not possible to choose proper (standard or inner) interval arithmetic operation.

Balanced random interval arithmetic proposed in (Žilinskas and Bogle, 2004) extending the ideas of (Alt and Lamotte, 2001), is obtained by choosing standard and inner interval operations at each step of the computation randomly with the predefined probabilities of standard and inner operations. A number of sample intervals are evaluated using balanced random interval arithmetic. It is assumed that the distributions of centres and radii of the evaluated balanced random intervals are normal and folded normal respectively. The range of values of function in the defined region is estimated using the mean values $(\mu)$ and the standard deviations $(\sigma)$ of centres and radii of the evaluated balanced random intervals:

$$
\left[\mu_{\text {centres }} \pm\left(3.0 \sigma_{\text {centres }}+\mu_{\text {radii }}+3.0 \sigma_{\text {radii }}\right)\right] .
$$

If the estimated range exceed the standard interval bounds for values of function in the same region, the evaluated interval is intersected with the standard interval while not reducing the probability that the estimated range contains all the values of function in the region.

The result of balanced random interval arithmetic is equal to the result of standard interval arithmetic when the predefined probability of standard interval operations is equal to 1 and the probability of inner interval operations is equal to 0 . The result of balanced 
random interval arithmetic is equal to the result of inner interval arithmetic when the predefined probability of standard interval operations is equal to 0 and the probability of inner interval operations is equal to 1 . Balanced random interval arithmetic provides wider or narrower ranges depending on the predefined probabilities. The values used for the predefined probabilities depend on the balance required between tightness of resulting intervals and the probability that resulting intervals contain all the values of function.

The disadvantage of balanced random interval arithmetic is that computations with this arithmetic use more calculations than with standard interval arithmetic, because the number of balanced random intervals should be evaluated. When 30 samples are used, balanced random interval arithmetic is more than 31 times more expensive than standard interval arithmetic. Another disadvantage is the assumption that distributions of centres and radii of the evaluated balanced random intervals are normal and folded normal respectively. The assumption is not true when computations involve small number of arithmetic operations, what was shown in (Žilinskas and Bogle, 2003) for simple mathematical global optimization test functions. To overcome these disadvantages the ranges of values of function should be estimated using smaller number of sample intervals (one or two) and estimation should not be based on the assumption of normal distributions.

\section{New Ways of Range Estimation Based on Interval Arithmetic}

In this paper we propose new ways to estimate range of values of function from standard and sometimes inner interval arithmetic results. If it would be possible to get ranges similar to ranges produced by balanced random interval arithmetic, new ways would be preferable because of speed of computations.

Relative centres and radii of ranges of values of objective function for practical multidimensional scaling problem in 100 random multidimensional intervals estimated using balanced random interval arithmetic with different predefined probabilities are shown in the Fig. 1. Shown centres and radii are relative to centres and radii of standard and inner intervals. The relative value of 0.0 means that the centre or radius is equal to the centre or
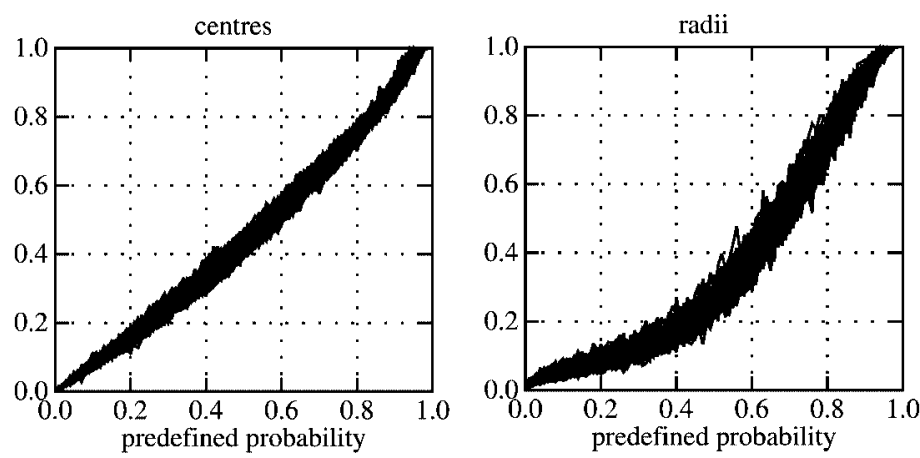

Fig. 1. Relative centres and radii of ranges estimated using balanced random interval arithmetic with different predefined probabilities. 
radius of inner interval. The relative value of 1.0 means that the centre or radius is equal to the centre or radius of standard interval.

The values of the centres and radii of ranges estimated using balanced random interval arithmetic move from the centres and radii of inner intervals toward the centres and radii of standard intervals when the predefined probability of standard interval operations increases. The relative values of the centres depend near linearly on the predefined probability. The relative values of the radii do not depend on the predefined probability linearly but they possibly may be modelled by linear dependency. Therefore a new estimate of range of values of function is defined as the weighted mean of resulting standard and inner intervals. Let us call it balanced interval arithmetic. Predefined coefficient $p c$ defines balance between standard and inner resulting intervals:

$$
p c \times \underline{\bar{f}}(\underline{\bar{X}})+(1-p c) \times \underline{f}_{i n}(\underline{\bar{X}}) .
$$

Other way to estimate range of values of function using standard and inner interval arithmetic is to balance standard and inner intervals in every interval operation:

$$
\underline{\bar{x}} \circ_{b} \underline{\bar{y}}=p c \times(\underline{\bar{x}} \circ \underline{\bar{y}})+(1-p c) \times\left(\underline{\bar{x}} \circ_{i n} \underline{\bar{y}}\right) .
$$

It is possible to estimate range of values of function tighter than standard interval from standard interval arithmetic only, if the centres of standard intervals are left unchanged and the radii are scaled by predefined coefficient $p c$. Let us call it scaled interval arithmetic:

$$
[(\underline{f}(\underline{\bar{X}})+\bar{f}(\underline{\bar{X}})) / 2 \pm p c \times(\bar{f}(\underline{\bar{X}})-\underline{f}(\underline{\bar{X}})) / 2] .
$$

Similarly as with balanced in every operation interval arithmetic, it is possible to scale the radii of standard intervals in every operation:

$$
\underline{\bar{x}} \circ s \underline{\bar{y}}=[(\underline{\underline{x} \circ \underline{\bar{y}}}+\underline{\overline{\bar{x}} \circ \underline{\bar{y}}}) / 2 \pm p c \times(\underline{\overline{\bar{x}} \circ \underline{\bar{y}}}-\underline{\underline{\bar{x}}} \circ \underline{\bar{y}}) / 2] .
$$

Balanced interval arithmetic, balanced in every operation interval arithmetic, scaled interval arithmetic and scaled in every operation interval arithmetic provide wider or narrower ranges depending on the predefined coefficient. The values used for the predefined coefficient depend on the balance required between tightness of resulting intervals and the probability that resulting intervals contain all the values of function. When the predefined coefficient is equal to 1 , the results of balanced interval arithmetic, balanced in every operation interval arithmetic, scaled interval arithmetic and scaled in every operation interval arithmetic are equal to the results of standard interval arithmetic. When the predefined coefficient is equal to 0 , the results of balanced interval arithmetic and balanced in every operation interval arithmetic are equal to the results of inner interval arithmetic, and the results of scaled interval arithmetic and scaled in every operation interval arithmetic are intervals with zero width and centres equal to the centres of resulting standard intervals. 


\section{Experimental Investigation of Proposed Estimates of Ranges}

Proposed estimates of ranges and balanced random interval arithmetic have been implemented in $\mathrm{C}++$ modifying a $\mathrm{C}++$ interval library filib++ (Lerch et al., 2001). It has been shown in (Žilinskas, 2005) that this library is most fast and accurate of freely available libraries implementing interval arithmetic. Moreover it is editable differently from integrated interval arithmetic library (SUN Microsystems, 2001) for the SUN Forte Compiler.

The ranges of values of functions in 1000 random regions have been estimated using proposed ways with different values of predefined coefficient and using balanced random interval arithmetic with different values of predefined probability of standard interval arithmetic operations.

Two criteria have been used in comparison of estimates of ranges: the success rate and the mean ratio of widths of estimated ranges and bounds evaluated using standard interval arithmetic. The success rate and the mean ratio for every function and every way to estimate ranges have been evaluated depending on the value of predefined coefficient or probability.

The success rate shows what part of ranges of values of given function over random regions is estimated successfully using given way with given predefined coefficient or predefined probability. Successful estimation means estimation of ranges which enclose all values of function in the region. As the exact range of values of function in random region is not known, it is estimated using random sampling. Values of function at 2000 random points uniformly distributed in the region are evaluated. Successful estimation is enclosure of these values. The success rate of 1.0 means that all ranges are estimated successfully and the success rate of 0.0 means that no ranges are estimated successfully. When estimated ranges for values of function are used in global optimization, the success rate determines the reliability of the global optimization algorithm. The algorithm is more reliable when the success rate is higher.

The mean ratio of widths shows how estimated ranges are tighter than bounds evaluated using standard interval arithmetic. As estimated ranges can not be wider than bounds evaluated using standard interval arithmetic, the mean ratio of 1.0 means that the widths of estimated ranges are equal to the widths of bounds evaluated using standard interval arithmetic. When estimated ranges of values of function are used in global optimization, the mean ratio of widths determines the speed of the global optimization algorithm. The mean ratio is smaller when estimated ranges are tighter and subregions are discarded earlier. So, the algorithm is faster when the mean ratio is smaller. The influence of tightness of bounds to speed of the algorithms for interval global optimization is experimentally investigated in (Žilinskas and Žilinskas, 2005a).

The success rate and mean ratio of widths of estimated ranges of values of multidimensional scaling function depending on the value of predefined probability or coefficient are shown in the Fig. 2. In this and in later figures, the solid line represents the criteria for balanced random interval arithmetic (1), dashed line - balanced interval arithmetic (2), dot dash - balanced in every operation interval arithmetic (3), dotted - scaled interval arithmetic (4), and dot dot dash - scaled in every operation interval arithmetic (5). The 

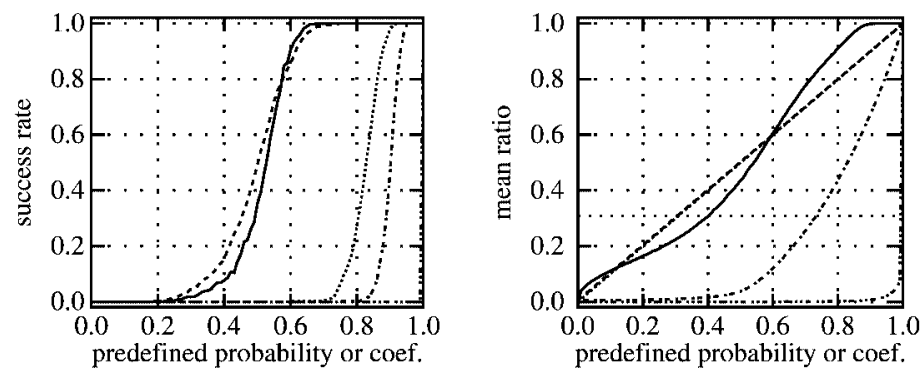

Fig. 2. Success rate and mean ratio of widths of estimated ranges of multidimensional scaling function depending on the value of predefined probability or coefficient.

mean ratio of widths of ranges of values of function estimated using random sampling and widths of bounds evaluated using standard interval arithmetic is shown as horizontal line similar to grid line. It is approximately 0.3 for multidimensional scaling function.

The estimation of ranges is more reliable when the success rate grows earlier, therefore it is more reliable when the curve representing the success rate is higher. The ranges are tighter when the mean ratio of widths grows later, therefore the estimated ranges are more tighter when the curve representing the mean ratio is lower. However both criteria depend on the predefined probability or coefficient and are related to each other. The relationship between the success rate and mean ratio of widths of estimated ranges shown in the Figs. 3 and 4 is more informative. The estimation is more reliable with tighter ranges when the curve representing it is higher. However curves of dependencies of the success rate and mean ratio on the predefined probability or coefficient are also very important because curve of relationship hides the case when criteria increase rapidly together making the use of range estimation difficult practically, what is the case for scaled in every operation interval arithmetic for multidimensional scaling function in the Fig. 2. However because of the shortage of space we will present only curves of the relationships in this paper. The mean ratio of widths of ranges of values of function estimated using random sampling and widths of bounds evaluated using standard interval arithmetic is shown as vertical line similar to grid line in the Figs. 3 and 4.

The relationship between the success rate and mean ratio of widths of estimated ranges of the function of practical multidimensional scaling problem is shown in the Fig. 3a. For this function balanced random interval arithmetic provides more reliability with tighter ranges than the proposed estimates, but balanced interval arithmetic provides very similar results. Ranges estimated using balanced random interval arithmetic and balanced interval arithmetic enclose values of function at random points when are $30 \%$ tighter than bounds evaluated using standard interval arithmetic. Results of scaled interval arithmetic and balanced in every operation interval arithmetic are worse and scaled in every operation interval arithmetic is not promising for estimation of ranges for this function.

The relationship between the success rate and mean ratio of widths of estimated ranges for the function of practical separation problem is shown in the Fig. 3b. For this function all ways to estimate ranges provide similar results. Estimated ranges enclose 


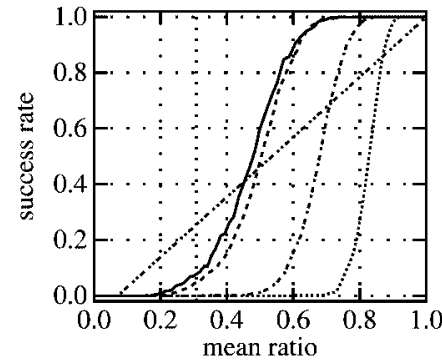

a) multidimensional scaling

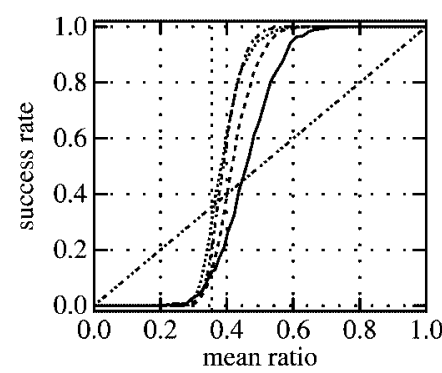

c) Paviani

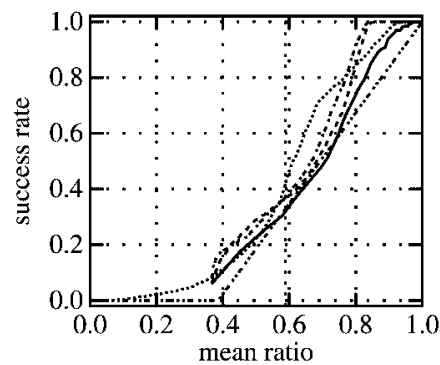

e) Six Hump Camel Back

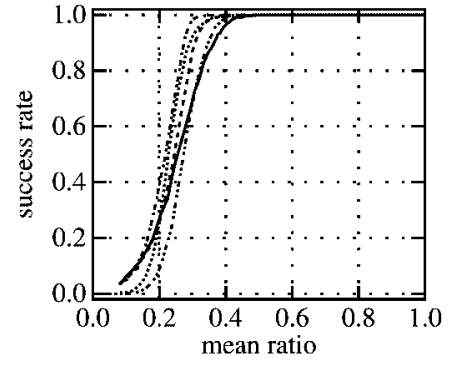

b) separation

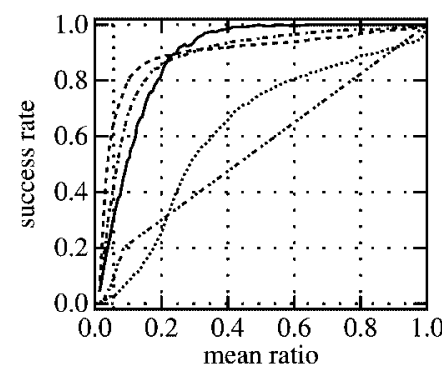

d) Goldstein and Price

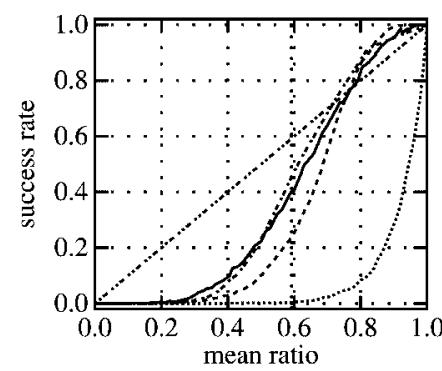

f) Shekel 5

Fig. 3. Relationship between the success rate and mean ratio of widths of estimated ranges.

values of function at random points when are $60 \%$ tighter than bounds evaluated using standard interval arithmetic. For this function ranges estimated using balanced in every operation interval arithmetic, scaled interval arithmetic and balanced interval arithmetic are a little bit better than using balanced random interval arithmetic and scaled in every operation interval arithmetic.

The relationship between the success rate and mean ratio of widths of estimated ranges of Paviani mathematical test function is shown in the Fig. 3c. For this function ranges estimated using balanced in every operation interval arithmetic, scaled interval arithmetic and balanced interval arithmetic are most promising. Ranges estimated using these ways enclose values of function at random points when are $40 \%$ tighter than bounds evaluated using standard interval arithmetic. Results of balanced random arithmetic are a little bit worse. Ranges estimated using this way enclose values of function at random 


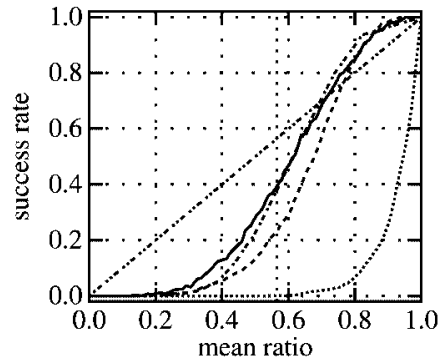

a) Shekel 7

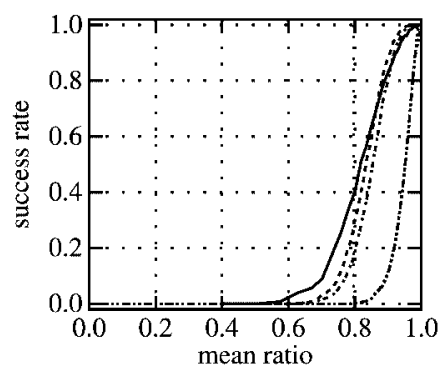

c) Levy 4

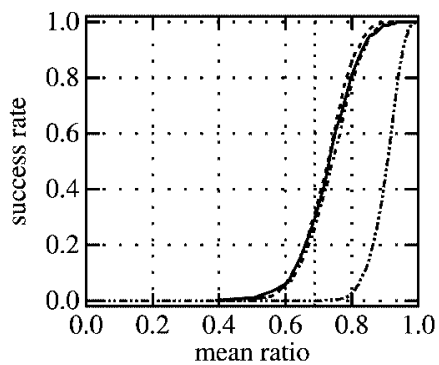

e) Levy 6

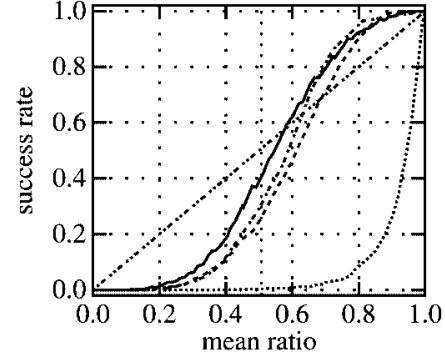

b) Shekel 10

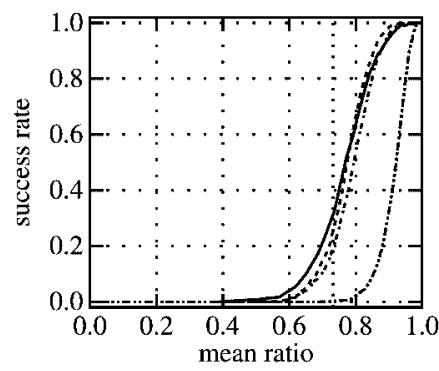

d) Levy 5

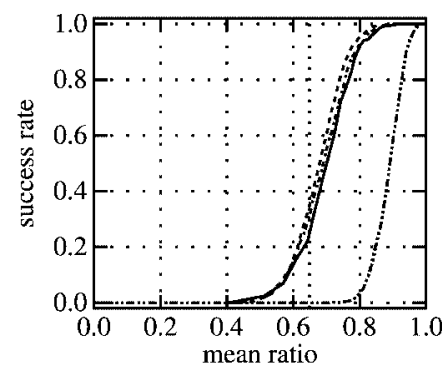

f) Levy 7

Fig. 4. Relationship between the success rate and mean ratio of widths of estimated ranges.

points when are $30 \%$ tighter than bounds evaluated using standard interval arithmetic. Scaled in every operation interval arithmetic is not promising for estimation of ranges of this function.

The relationship between the success rate and mean ratio of widths of estimated ranges of Goldstein and Price mathematical test function is shown in the Fig. 3d. Only ranges estimated using balanced random interval arithmetic are promising for this function. Ranges estimated using this way enclose values of function at random points when are $40 \%$ tighter than bounds evaluated using standard interval arithmetic.

The relationship between the success rate and mean ratio of widths of estimated ranges of Six Hump Camel Back mathematical test function is shown in the Fig. 3e. Ranges estimated with balanced interval arithmetic and balanced in every operation interval arithmetic are promising for this function. Ranges estimated using these ways enclose 
values of function at random points when are 15\% tighter than bounds evaluated using standard interval arithmetic.

The relationships between the success rate and mean ratio of widths of estimated ranges for Shekel 5, 7 and 10 mathematical test functions are shown in the Figs. 3f, 4a and $4 \mathrm{~b}$. Ranges estimated with balanced interval arithmetic and balanced random interval arithmetic are promising for these functions. Ranges estimated using these ways enclose values of functions at random points when are $10 \%$ tighter than bounds evaluated using standard interval arithmetic. Scaled interval arithmetic and scaled in every operation interval arithmetic are not promising. Curves of dependencies of the success rate and mean ratio on the predefined probability or coefficient show that balanced in every operation interval arithmetic is not promising too.

The relationships between the success rate and mean ratio of widths of estimated ranges for Levy 4, 5, 6 and 7 mathematical test functions are shown in the Figs. 4c, 4d, 4e and 4f. Ranges estimated with balanced interval arithmetic, balanced in every operation interval arithmetic and balanced random interval arithmetic are promising for these functions. Ranges estimated using these ways enclose values of functions at random points when are 5-15\% tighter than bounds evaluated using standard interval arithmetic. Scaled in every operation interval arithmetic is not promising. Curves of dependencies of the success rate and mean ratio on the predefined probability or coefficient show that scaled interval arithmetic is not promising too.

Results of the experiments show that balanced interval arithmetic is most promising of the proposed ways for estimation of ranges for functions. Ranges estimated using this way compete with ranges estimated using balanced random interval arithmetic and sometimes are even better. Balanced in every operation interval arithmetic is a little bit less promising, scaled interval arithmetic is even less promising, and scaled in every operation interval arithmetic is not promising. It is important to note that proposed ways require much less computations than balanced random interval arithmetic.

\section{Conclusions}

To overcome disadvantages of interval arithmetic and balanced random interval arithmetic new ways to estimate ranges of values of functions from standard and inner interval arithmetic have been proposed. Using the proposed ways ranges of values of mathematical test functions for global optimization and of functions for practical problems have been estimated and compared. Results of the experiments show that balanced interval arithmetic is most promising of the proposed ways for estimation of ranges of values of functions. Ranges estimated using balanced interval arithmetic compete with ranges estimated using balanced random interval arithmetic and sometimes are even better, while they require less computations than ranges estimated using balanced random interval arithmetic and are not based on assumption of normal distributions. It is promising to use proposed balanced interval arithmetic in interval global optimization. 


\section{Acknowledgment}

The research is supported by the NATO Reintegration grant CBP.EAP.RIG.981300.

\section{References}

Alt, R., and J.L. Lamotte (2001). Experiments on the evaluation of functional ranges using random interval arithmetic. Mathematics and Computers in Simulation, 56(1), 17-34.

Csendes, T. (1998). Optimization methods for process network synthesis - a case study. In: C. Carlsson and I. Eriksson (Eds.), Global and Multiple Criteria Optimization and Information Systems Quality. Abo Academy, Turku. pp. 113-132.

Hansen, E., and G. Walster (2003). Global Optimization Using Interval Analysis, 2nd Edition. Marcel Dekker, New York.

Johnson, N.L., and S. Kotz (1994-1995). Continuous Univariate Distributions, 2nd Edition. John Wiley, New York.

Kreinovich, V., V. Nesterov and N. Zheludeva (1996). Interval methods that are guaranteed to underestimate and the resulting new justification of Kaucher arithmetic. Reliable Computing, 2(2), 119-124.

Lerch, M., G. Tischler, J.W. von Gudenberg, W. Hofschuster and W. Krämer (2001). The Interval Library filib++ 2.0 - Design, Features and Sample Programs. Preprint 2001/4, Universität Wuppertal.

Madsen, K., and J. Žilinskas (2000). Testing Branch-and-Bound Methods for Global Optimization. Technical report 05/2000, Technical University of Denmark.

Moore, R.E. (1966). Interval Analysis. Prentice-Hall.

SUN Microsystems (2001). C++ Interval Arithmetic Programming Reference. Forte Developer 6 Update 2 (Sun WorkShop 6 update 2).

Žilinskas, J. (2005). Comparison of packages for interval arithmetic. Informatica, 16(1), 145-154.

Žilinskas, J., and I.D.L. Bogle (2003). Evaluation ranges of functions using balanced random interval arithmetic. Informatica, 14(3), 403-416.

Žilinskas, J., and I.D.L. Bogle (2004). Balanced random interval arithmetic. Computers and Chemical Engineering, 28(5), 839-851.

Žilinskas, A., and J. Žilinskas (2005a). On efficiency of tightening bounds in interval global optimization. Lecture Notes in Computer Science, 3732, 197-205.

Žilinskas, A., and J. Žilinskas (2005b). On underestimating in interval computations. BIT Numerical Mathematics, 28(5), 839-851.

J. Žilinskas is a senior researcher in Systems Analysis Department at the Institute of Mathematics and Informatics, Lithuania. He studied informatics at Kaunas University of Technology receiving his $\mathrm{PhD}$ in 2002. His research interests include global optimization and parallel computing.

\section{Nauji intervalu aritmetika pagristi funkciju reikšmiu rèžiu ivertinimo būdai}

\section{Julius ŽILINSKAS}

Straipsnyje pasiūlyti nauji funkciju reikšmiu rèžiu ivertinimo iš standartinès ir vidinès intervalu aritmetikos būdai. Ivertinti ir palyginti pasiūlyti matematiniu globalios optimizacijos testavimo funkcijų ir praktiniu globalios optimizacijos uždavinių tikslo funkcijų reikšmiu rěžių įverčiai. Eksperimento rezultatai rodo, kad pasiūlyta balansuojama intervalų aritmetika gali būti naudojama intervalinėje globalioje optimizacijoje. 